\title{
High Energy Phenomena at the Center of our Galaxy
}

\section{Andrea Goldwurm*}

AstroParticule et Cosmologie

E-mail: andrea.goldwurm@cea.fr

I will review the recent results on the high-energy phenomena occuring in the region of the Galactic Center by first describing the structure and the components of this fascinating area of the sky and then reporting the main discoveries that have been obtained in X-rays and gamma-rays. Several of these results indicate that the Galactic Center was the site of a powerful activity in the past, possibly driven by the central supermassive black hole, $\operatorname{Sgr} A^{*}$, that, today, appears very dim and quiet in contrast with the exuberant activity displayed by other galactic nuclei. I will try to put these results in contest with the general picture we have from the observations of the nuclei of external galaxies to see if this can lead to some new insights on the impact of the galactic supermassive black hole on its close environment and on the whole Galaxy.

Frontiers of Fundamental Physics 14 - FFP14,

15-18 July 2014

Aix Marseille University (AMU) Saint-Charles Campus, Marseille

* Speaker. 